\title{
The NEMO educational kit
}

H. Thienpont, J. Mohr, M. Kujawinska, M. Taghizadeh, A. Waddie, et al.

H. Thienpont, J. Mohr, M. Kujawinska, M. R. Taghizadeh, A. J. Waddie, T. Mappes, F. Wyrowski, E. Stijns, "The NEMO educational kit," Proc. SPIE 9665, Tenth International Topical Meeting on Education and Training in Optics and Photonics, 96651S (3 June 2007); doi: 10.1117/12.2207476

Event: Tenth International Topical Meeting on Education and Training in Optics and Photonics, 2007, Ottawa, Ontario, Canada 
The NEMO educational kit

H. Thienpont ${ }^{1}$, J. Mohr ${ }^{2}$, M. Kujawinska ${ }^{3}$, M. R. Taghizadeh ${ }^{4}$, A. J. Waddie ${ }^{4}$, T. Mappes $^{2}$, F. Wyrowski ${ }^{5}$, E. Stijns ${ }^{1}$

${ }^{1}$ VUB Vrije Universiteit Brussel, IR-TONA, Brussel, Belgium;

${ }^{2}$ FZK Forschungszentrum Karlsruhe, Germany;

${ }^{3}$ MWUT Warsaw Univ of Technology, Warsaw, Poland;

${ }^{4}$ School of Engineering and Physical Sciences, Heriot-Watt University, Edinburgh EH14 4AS, UK

${ }^{5}$ LightTrans GmbH and University of Jena, Jena, Germany

\begin{abstract}
$\underline{\text { Abstract }}$
NEMO is the European "Network of Excellence on Micro-Optics". One of the objectives is to disseminate knowledge on micro-optics. Therefore NEMO plans to inform pupils about the crucial role of micro-optics This is done through the distribution of an educational kit to their physics/technology teachers. This kit has been realized through a cooperative action of different partners of the NEMO-network all over Europe. It contains a variety of replicated micro-optical refractive and diffractive components, and a semiconductor laser source. The kit is supplemented with a CD-ROM which explains the basic concepts and describes possible experiments and experimental setups. It contains also a computer tutorial which simulates the optical processes of image formation.

It is hoped that this will encourage interest in optics and, more generally, in science as an area of future study and as a possible career choice.

At the conference the realization and the lay out of the EduKit will be commented and a demonstration will be given.
\end{abstract}

Tenth International Topical Meeting on Education and Training in Optics and Photonics, edited by Marc Nantel, Proc. of SPIE Vol. 9665, 96651S · (c) 2007 SPIE, OSA, IEEE, ICO doi: $10.1117 / 12.2207476$ 


\section{Introduction}

NEMO is the "Network of Excellence in Micro-Optics" constructed under the European "Sixth Framework Programme". It aims at providing Europe with a complete Micro-Optics food-chain, by setting up centers for optical modeling and design, measurement and instrumentation, mastering, prototyping and replication, hybrid integration and packaging, reliability and standardization. More than 300 researchers from 30 groups in 12 countries participate in the project.

One of the objectives of NEMO is to spread excellence and disseminate knowledge on micro-optics and micro-photonics. Therefore NEMO plans to inform pupils, already from secondary school level onwards, about the crucial role of light and micro-optics and the opportunities this combination holds. This is be done through the distribution of a userfriendly and well illustrated educational kit to their physics/technology teachers. The latter contains a variety of replicated micro-optical refractive and diffractive components, fabricated with the aid of the network technology centers, a semiconductor laser source and a clear and instructive manual for basic experiments. The kit is packed in a box, paid by SPIE-Europe. It is self-consistent: schools with no optical instrumentation are able to use it for basic demonstrations. However it is also possible to use the elements in more complicated set-ups to be constructed by the users themselves.

\section{The realization of the Educational Kit}

The EduKit consists of a DVD-box with:

- a laserpointer

- a piece of plastic optical fibre

- a CD-ROM with the necessary explanation

- a plastic card with refractive and diffractive optical components.

Those components were designed in the group of Mo Taghizadeh in the Heriot-Watt University, Scotland, UK., and replicated by the group of Juergen Mohr in Karlsruhe, Germany ${ }^{(1)}$. The layout is shown below.

On a small plastic card (see figure 1) you find the different diffractive optical elements or DOEs, and also arrays of micro-lenses. 


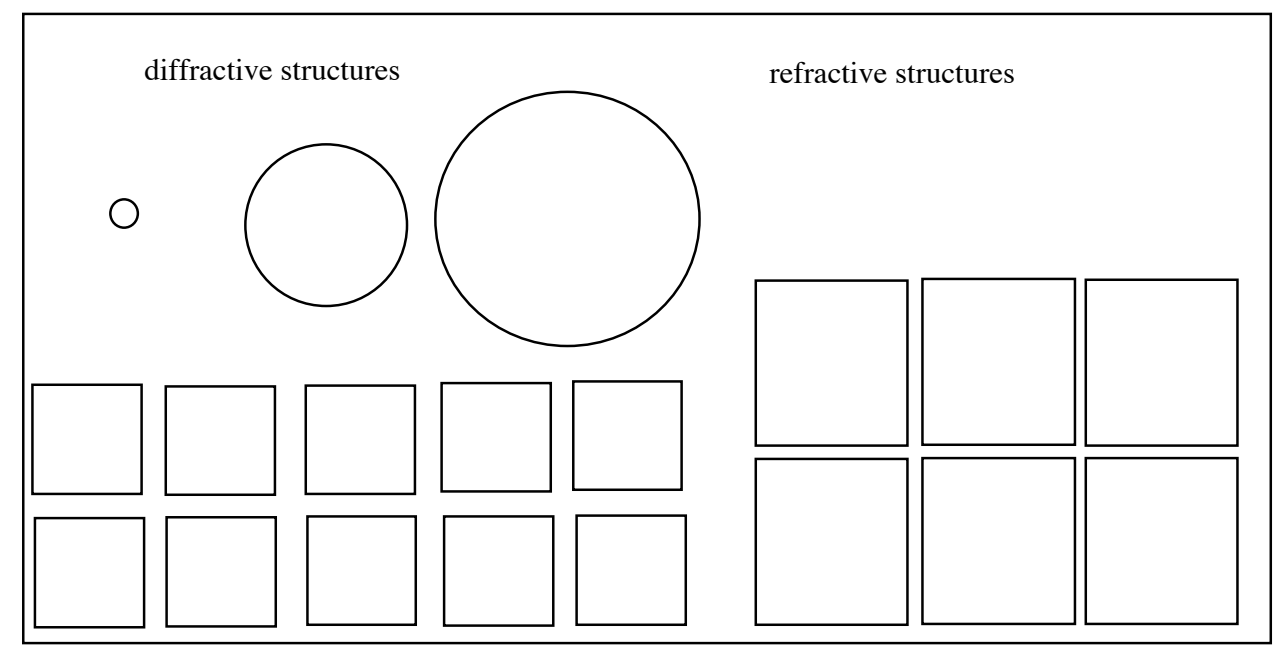

Figure 1: lay out of the plastic card with optical elements

On the left you find the diffractive elements or DOEs. There are two simple linear gratings, with a pitch of about 60 lines per millimeter (lpmm) respectively about 30 lpmm; they give a row of equidistant points on the projection screen.

The card contains also two crossed grating, once again with spatial frequencies of 60 respectively $30 \mathrm{lpmm}$; their far-field diffraction pattern consists of a regular square grid of points.

Next there is a set of PF-DOE's (= Pattern Forming Diffractive Optical Elements), which produces the following patterns:

- two array generators or "fan-out" elements which split the incident laser beam into a regular square grid of $4 \times 4$ respectively $8 \times 8$ points of equal intensity.

- there are also two flattop generator: one on-axis and one off-axis

- the next PF-DOE gives a square grid

- finally the last two FP-DOE give the logos of the NEMO network and a European flag.

Moreover on the card you find also three FZP or Fresnel Zone Plate's with focal distances of respectively 10,50 and $250 \mathrm{~mm}$.

Finally the refractive elements (on the right side of the card) are arrays of very small lenses, so-called micro-lenses: two square arrays of micro-lenses, two arrays of cylindrical lenses, and two hexagonal arrays of micro-lenses. The distances between neighbouring lenses are either $0,1 \mathrm{~mm}$ or $0,05 \mathrm{~mm}$.

The companion CD-ROM gives not only information about the experiments to be performed with the Kit (see next section), but gives also background information about the NEMO-network, and also examples of the uses of diffractive elements in actual instrumentation. Moreover you find explanations about the basic concepts of light rays, waves, diffraction and interference.

On the CD-ROM you also find a "tutorial" which simulates the basic experiment of diffraction, i.c. Young's interference experiment; you can change the distance between the slits and/or the wavelength, and look at the changing image on the screen. There is also a computer program included, realized by $\mathrm{LTG}^{(2)}$, which calculates the light distribution behind the diffractive elements as a function of variable parameters. 


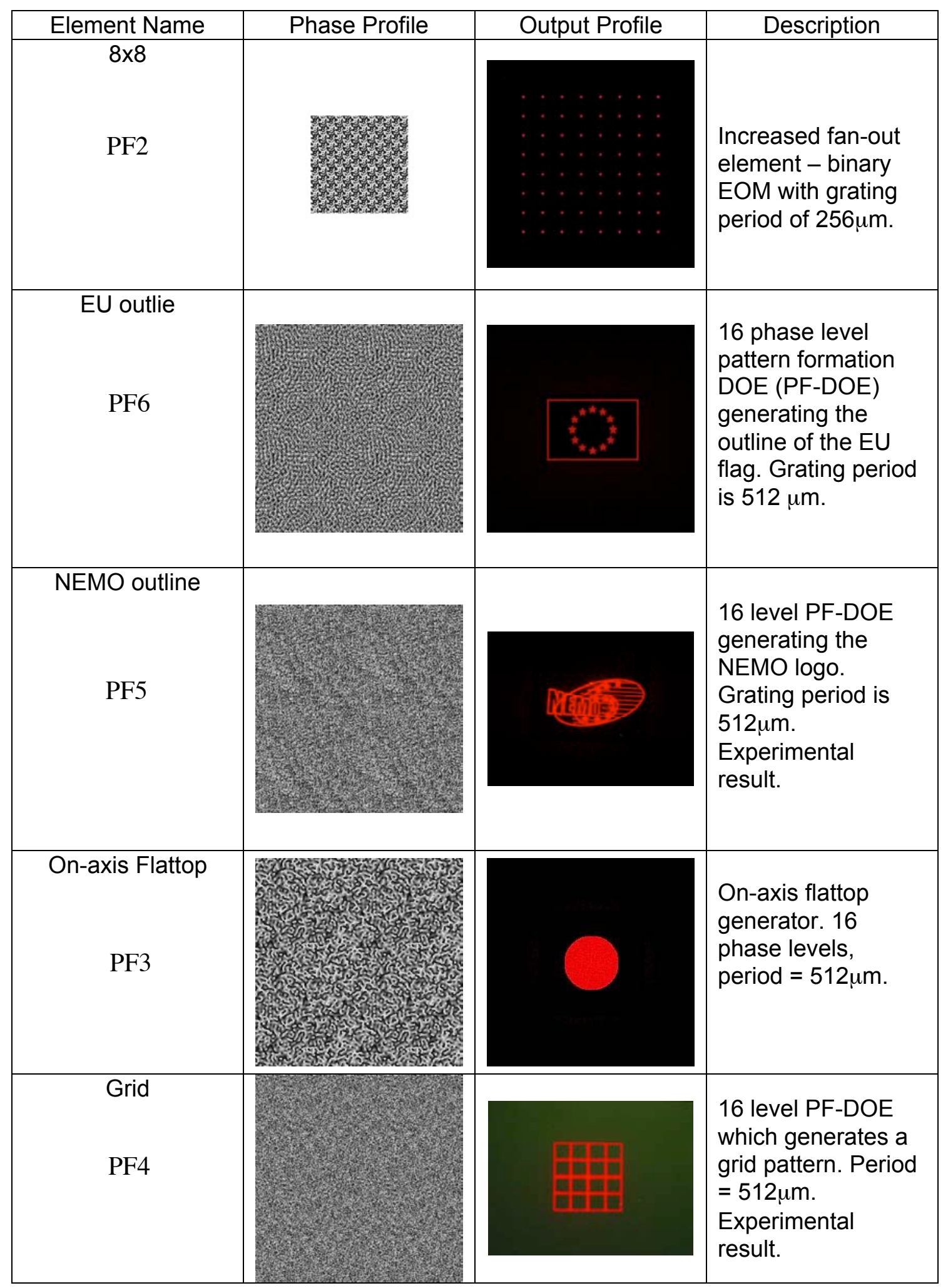

Figure 2: picture of some DOEs (left) and their projection on a distant screen (right). 


\section{The use of it}

In the most simple experiment, you shine directly with a laser (-pointer) through the diffractive elements, and look at the image on a distant screen, see figure 3.

The included CD-ROM also explains which images you can expect for each of the elements, when using the plastic card in this simple projection set up.

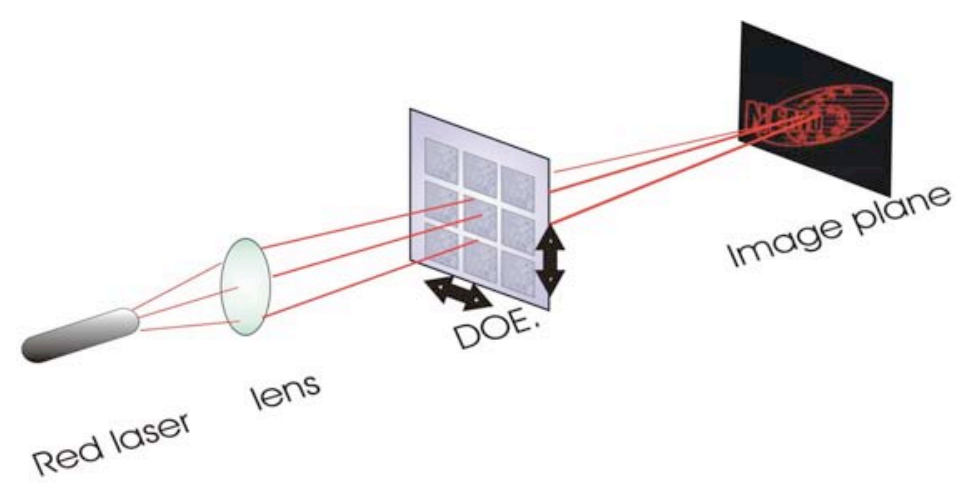

Figure 3: realization of the far-field diffraction pattern by simple projection

However, the same plastic card with DOEs can also be used for much more complicated set ups, if you have more optical hardware available. You can experimentally produce a Fourier transform, or even build a 4-f processor for optical filtering. It is clear that the latter experiments require a deeper theoretical knowledge, and so they are reserved for higher schools or even universities.

There is also a section included on laser safety.

\section{How is it distributed and used?}

The EduKit was designed and produced within the framework of the NEMO-network, a project sponsored by the European Commission. This sponsoring allows for a free distribution of the EduKit to teachers of secondary schools in Europe. It is expected that they use it in their lessons on physics or technology. Afterwards, they fill in an "evaluation form", so that a next and improved/extended edition can be produced.

More information is to be found at www.micro-optics.org .

\section{References}

${ }^{(1)}$ A. J. Waddie, M. R. Taghizadeh, J. Mohr, V. Piotter, Ch. Mehne, A. Stuck, E. Stijns, H. Thienpont: Design, Fabrication and Replication of Micro-Optical Components for Educational Purposes within the Network of Excellence in Micro-Optics (NEMO)

Proceedings of the SPIE (Photonics Europe 2006), Vol. 6185: Micro-Optics, VCSEL and photonics interconnects, pp 91-98, 2006

${ }^{(2)}$ LightTrans GmbH, Jena, Germany: (www.lighttrans.com) 